\title{
Fatigue in primary Sjögren's syndrome (pSS) is associated with lower levels of proinflammatory cytokines: a validation study
}

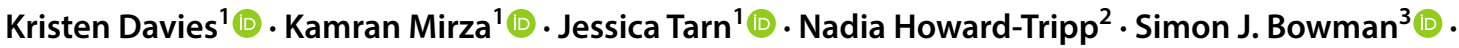 \\ Dennis Lendrem ${ }^{1,2,4} \odot$. UK Primary Sjögren's Syndrome Registry $\cdot$ Wan-Fai $\mathrm{Ng}^{1,2,4}([)$
}

Received: 9 April 2019 / Accepted: 18 June 2019 / Published online: 27 June 2019

(c) The Author(s) 2019

\begin{abstract}
Primary Sjögren's syndrome (pSS) is a chronic autoimmune rheumatic disease with symptoms including dryness, fatigue, and pain. The previous work by our group has suggested that certain proinflammatory cytokines are inversely related to patient-reported levels of fatigue. To date, these findings have not been validated. This study aims to validate this observation. Blood levels of seven cytokines were measured in 120 patients with pSS from the United Kingdom Primary Sjögren's Syndrome Registry and 30 age-matched healthy non-fatigued controls. Patient-reported scores for fatigue were classified according to severity and compared to cytokine levels using analysis of variance. The differences between cytokines in cases and controls were evaluated using Wilcoxon test. A logistic regression model was used to determine the most important identifiers of fatigue. Five cytokines, interferon- $\gamma$-induced protein-10 (IP-10), tumour necrosis factor- $\alpha$ (TNF $\alpha$ ), interferon- $\alpha$ (IFN $\alpha)$, interferon- $\gamma$ (IFN- $\gamma)$, and lymphotoxin- $\alpha(\mathrm{LT}-\alpha)$ were significantly higher in patients with $\mathrm{pSS}(n=120)$ compared to non-fatigued controls $(n=30)$. Levels of two proinflammatory cytokines, TNF- $\alpha(p=0.021)$ and LT- $\alpha(p=0.043)$, were inversely related to patient-reported levels of fatigue. Cytokine levels, disease-specific and clinical parameters as well as pain, anxiety, and depression were used as predictors in our validation model. The model correctly identifies fatigue levels with $85 \%$ accuracy. Consistent with the original study, pain, depression, and proinflammatory cytokines appear to be the most powerful predictors of fatigue in pSS. TNF- $\alpha$ and LT- $\alpha$ have an inverse relationship with fatigue severity in pSS challenging the notion that proinflammatory cytokines directly mediate fatigue in chronic immunological conditions.
\end{abstract}

Keywords Fatigue $\cdot$ Primary Sjögren’s syndrome $\cdot$ Proinflammatory $\cdot$ Cytokines

This study was previously presented and published as an American College of Rheumatology (ACR) abstract at the 2017 ACR Conference in San Diego, California. Abstract can be found here: https://acrabstracts.org/abstract/fatigue-in-primary-sjogrens-syndr ome-pss-is-associated-with-lower-levels-of-proinflammatory -cytokines-a-validation-study/.

The members of UK Primary Sjögren's Syndrome Registry are listed in the Acknowledgements section.

Electronic supplementary material The online version of this article (https://doi.org/10.1007/s00296-019-04354-0) contains supplementary material, which is available to authorized users.

Kristen Davies

kristen.davies@newcastle.ac.uk; kristen.davies4@gmail.com

Extended author information available on the last page of the article

\section{Introduction}

Fatigue is a complex and disabling symptom affecting between 22 and $30 \%$ of the general population resulting in reduced quality of life and associated with substantial economic cost [1-5]. It is a prominent feature of numerous chronic diseases, particularly rheumatic diseases such as primary Sjögren's syndrome (pSS) [5, 6].

The aetiology of fatigue is complex and likely multifactorial, with both biological and psychosocial elements contributing towards the perception of fatigue. Regarding the biological basis of fatigue, proinflammatory cytokines may play a central role [7]. Fatigue is frequently observed in immune-mediated inflammatory conditions and following infections, as part of a constellation of symptoms termed 'sickness behaviour'. An adaptive response to infection, sickness behaviour minimises energy expenditure when an 
organism is in a weakened state following an infection and resolves with the resolution of inflammation [7].

Research previously performed by our group investigated the relationship between proinflammatory cytokines and fatigue in pSS [8]. Howard-Tripp et al. found 14 cytokines to be significantly higher in patients with pSS compared to non-fatigued controls. When patients with pSS were grouped according to fatigue severity, an unexpected inverse relationship was found between fatigue scores and four proinflammatory cytokines, IP-10, TNF- $\alpha$, LT- $\alpha$, and IFN- $\gamma$. Considering the role cytokines play in the development of the initial inflammatory response, our group postulated that a potentially maladaptive immune response may contribute to the maintenance of persistent fatigue in a chronic inflammatory state as observed in conditions such as pSS. A logistic regression model was created using the cytokines and clinical and disease-specific parameters, which was able to predict fatigue levels with reasonable accuracy (67\%). Similar accuracy was reported using only IP-10 and IFN- $\gamma$, along with depression and pain scores.

This study aims to validate the previously reported paradoxical observation of an inverse association between proinflammatory cytokines and patient-reported fatigue scores. We measured the serum levels of seven proinflammatory cytokines - interferon- $\gamma$-induced protein 10 (IP-10), tumour necrosis factor- $\alpha$ (TNF- $\alpha$ ), lymphotoxin- $\alpha$ (LT- $\alpha)$, interferon- $\gamma(\mathrm{IFN}-\gamma)$, interferon- $\alpha$ (IFN- $\alpha)$, interleukin12p70 (IL-12p70), and interleukin-17 (IL-17). Six of these were reported to be significantly higher in pSS patients [8] including four demonstrating an inverse relationship with fatigue levels. In addition, while IFN- $\alpha$ was not found to be significantly higher in pSS patients, it has been well documented as a potential inducer of fatigue [9].

\section{Methods}

\section{Experimental design}

The objective of this study was to analyse proinflammatory cytokine and fatigue levels in patients with pSS to validate the previously observed relationship between cytokines and fatigue in pSS. Similar to the original study, we used clinical and biological data to ascertain the most important predictors of fatigue within this patient group.

\section{Study population}

An independent cohort of patients were selected from the United Kingdom Primary Sjögren's Syndrome Registry (UKPSSR) [10]. The UKPSSR holds clinical, haematological, and demographic data on over 900 patients with pSS and 350 healthy controls across 37 centres in the UK. All patients on UKPSSR fulfil the American European Consensus Group criteria for classification of pSS. This study randomly selected 30 blood samples each from female patients with pSS with either minimal, mild, moderate, or severe fatigue, using a random number generator, for a total of 120 patients. Thirty non-fatigued healthy controls from the UKPSSR were also selected. The North West Research Ethics Committee granted the ethical approval for this study.

\section{Clinical parameters}

The following clinical parameters were collected contemporaneously as the biological samples for this study. Fatigue severity was measured using the Profile of Fatigue (PROF) Questionnaire which has been validated for pSS [11]. Physical fatigue was scored on a scale of 0-7 to group patients into minimal (0-1), mild (2-3), moderate (4-5), and severe (6-7) fatigue groups. Controls were screened for the presence of fatigue using a self-reported questionnaire. None of the controls reported the presence of fatigue or autoimmune disease. Anxiety and depression were measured using the Hospital Anxiety and Depression Score [12].

Disease-specific measures such as the EULAR Sjögren's Syndrome Disease Activity Index (ESSDAI) and the EULAR Sjögren's Syndrome Patient-Reported Index (ESSPRI), Schirmer's test, unstimulated oral salivary flow, and the EULAR Sicca Score were also included [13, 14].

Clinical laboratory data including white cell count (WCC), lymphocytes, neutrophils, haemoglobin, erythrocyte sedimentation rate (ESR), and C-reactive protein (CRP) were measured by the NHS laboratory of the recruiting centre within a day of sample collection.

\section{Cytokine measurement}

Proinflammatory cytokines were measured using biobanked serum samples from the UKPSSR. Seven cytokines were tested: Interferon- $\gamma$-induced protein 10 (IP-10), tumour necrosis factor- $\alpha$ (TNF- $\alpha$ ), lymphotoxin- $\alpha$ (LT- $\alpha)$, interferon- $\gamma($ IFN- $\gamma)$, interferon- $\alpha$ (IFN- $\alpha)$, interleukin12p70 (IL-12p70), and interleukin-17 (IL-17). Six cytokines were analysed with cytometric bead array and IL-17 was measured using enzyme-linked immunosorbent assay as previously described [9].

\section{Statistical analysis}

Patient demographic data and clinical data are presented using median and IQR. Statistical significance was determined using Kruskal-Wallis test. Cytokine levels were log transformed prior to analysis of variance, which was used to examine the relationship between the cytokine level and the corresponding fatigue score. 
Ordinal logistic regression analysis was used to model fatigue levels using all cytokines, WCC, lymphocytes, neutrophils, ESR, CRP, ESSDAI, and dryness scores, as well as depression, pain, and anxiety scores. The tests performed on this data set were robust to FDR multiple test correction.

All statistical analyses and graphical visualizations were performed using R version 3.1.1 and SAS JMP Pro (Version 14) Statistical Data Visualisation software $[15,16]$.

\section{Results}

\section{Study population}

Patients with pSS were stratified into four groups according to their fatigue levels as previously described [9]. All patients were female and predominantly Caucasian. When comparing fatigue groups, there were statistically significant differences between ESSDAI, ESSPRI, EULAR-SS, WCC, lymphocytes, and BMI (Table 1). Age, disease, and symptom duration were not significantly different between groups. Anti-Ro/La positivity and percentages of each group taking immune-altering medications did not differ significantly between groups.

\section{Cytokine differences between patients with pSS and healthy controls}

All proinflammatory cytokines were higher in the pSS population than controls: five proinflammatory cytokines, IP-10, TNF $\alpha$, LT $\alpha$, IFN-y, and IFN $\alpha$, were significantly higher in patients with pSS compared to controls, and IL-12p70 was also close to statistical significance (see Fig. 1).

\section{Cytokine and fatigues scores in pSS}

All four of the cytokines evaluated in the original studyIP-10, IFN-y, TNF- $\alpha$, and LT- $\alpha$ - showed an inverse relationship with patient-reported levels of fatigue. This was statistically significant for TNF- $\alpha(p=0.021)$ and LT- $\alpha$ $(p=0.043)$. Overall, the previously reported trend of higher cytokine levels associated with reduced fatigue scores in pSS patients was replicated-see Fig. 2.
Table 1 Clinical summary for the four separate pSS fatigue groups demonstrating mean and standard deviation for key demographics, haematological, and clinical variables

\begin{tabular}{|c|c|c|c|c|c|}
\hline & Minimal & Mild & Moderate & Severe & $p$ value \\
\hline Age (years) & $53 \pm 13$ & $58 \pm 14$ & $60 \pm 12$ & $56 \pm 12$ & Ns \\
\hline Disease duration (years) & $4.4 \pm 5.5$ & $7.0 \pm 7.7$ & $4.3 \pm 4.9$ & $5.1 \pm 7.3$ & Ns \\
\hline Symptom duration (years) & $9 \pm 7$ & $12 \pm 9$ & $9 \pm 7$ & $9 \pm 8$ & Ns \\
\hline BMI $\left(\mathrm{kg} / \mathrm{m}^{2}\right)$ & $26 \pm 5$ & $24 \pm 4$ & $26 \pm 6$ & $30 \pm 7$ & 0.0017 \\
\hline$\%$ not taking immune-altering medications & 43 & 23 & 30 & 20 & Ns \\
\hline$\%$ on hydroxychloroquine & 33 & 43 & 40 & 46 & Ns \\
\hline$\%$ on prednisolone & 7 & 10 & 7 & 20 & Ns \\
\hline$\%$ on 'other' immune-altering medications & 13 & 24 & 33 & 14 & Ns \\
\hline ESSDAI & $2.8 \pm 2.9$ & $3.3 \pm 3.4$ & $3.4 \pm 3.7$ & $4.8 \pm 3.4$ & Ns \\
\hline ESSPRI & $2.6 \pm 1.6$ & $4.6 \pm 1.3$ & $5.8 \pm 1.6$ & $8.3 \pm 1.0$ & $<0.0001$ \\
\hline ESSPRI pain & $0.8 \pm 0.8$ & $3.4 \pm 0.8$ & $5.0 \pm 0.8$ & $6.5 \pm 0.5$ & $<0.0001$ \\
\hline ESSPRI dryness & $1.3 \pm 1.2$ & $3.5 \pm 0.9$ & $4.9 \pm 0.8$ & $6.3 \pm 0.7$ & $<0.0001$ \\
\hline EULAR-SS & $3.8 \pm 2.5$ & $5.4 \pm 2.3$ & $5.9 \pm 2.1$ & $8.1 \pm 1.6$ & $<0.0001$ \\
\hline HADS anxiety $(0-21)$ & $4.4 \pm 3.2$ & $8.3 \pm 3.9$ & $8.9 \pm 4.3$ & $10.5 \pm 5.2$ & $<0.0001$ \\
\hline HADS depression $(0-21)$ & $2.4 \pm 2.5$ & $4.9 \pm 3.1$ & $6.6 \pm 3.2$ & $10.6 \pm 4.2$ & $<0.0001$ \\
\hline $\mathrm{Ro}+/ \mathrm{La}+$ & 15 & 22 & 14 & 17 & Ns \\
\hline $\mathrm{Ro}+/ \mathrm{La}-$ & 8 & 5 & 6 & 7 & Ns \\
\hline Ro-/La+ & 1 & 1 & 0 & 0 & Ns \\
\hline Ro-/La- & 4 & 1 & 9 & 6 & Ns \\
\hline $\mathrm{Hb}(\mathrm{g} / \mathrm{dL})$ & $13 \pm 0.9$ & $13 \pm 0.9$ & $13 \pm 1.3$ & $13 \pm 1.2$ & Ns \\
\hline WCC $\left(\times 10^{9} / \mathrm{L}\right)$ & $5.0 \pm 1.3$ & $5.0 \pm 1.2$ & $5.6 \pm 2.0$ & $6.6 \pm 2.6$ & 0.0042 \\
\hline Neutrophil $\left(\times 10^{9} / \mathrm{L}\right)$ & $3.0 \pm 1.0$ & $3.1 \pm 0.9$ & $3.3 \pm 1.3$ & $3.7 \pm 1.6$ & Ns \\
\hline Lymphocyte $\left(\times 10^{9} / \mathrm{L}\right)$ & $1.4 \pm 0.6$ & $1.3 \pm 0.4$ & $1.6 \pm 0.6$ & $1.8 \pm 0.6$ & 0.0095 \\
\hline $\operatorname{ESR}(\mathrm{mm} / \mathrm{h})$ & $27 \pm 21$ & $22 \pm 18$ & $23 \pm 20$ & $24 \pm 23$ & Ns \\
\hline CRP (mg/L) & $1.7 \pm 5.2$ & $1.8 \pm 5.8$ & $2.2 \pm 3.0$ & $4.1 \pm 5.6$ & Ns \\
\hline $\operatorname{IgG}(\mathrm{mg} / \mathrm{dL})$ & $18 \pm 8$ & $16 \pm 9$ & $15 \pm 7$ & $14 \pm 5$ & Ns \\
\hline
\end{tabular}

Bold $p$ values indicate statistical significance $(<0.05)$ 
A

\begin{tabular}{|c|c|c|c|}
\hline \multirow[t]{2}{*}{ Cytokine } & Controls $n=\mathbf{3 0}$ & pSS cases $n=120$ & \multirow[t]{2}{*}{ p Value } \\
\hline & \multicolumn{2}{|c|}{$\begin{array}{c}\text { Median } \\
25^{\text {th }}, 75^{\text {th }} \text { centile }(\mathrm{pmol} / \mathrm{L})\end{array}$} & \\
\hline IP-10 & $\begin{array}{c}431.76 \\
251.44,601.49\end{array}$ & $\begin{array}{c}684.09 \\
468.86,1030.105\end{array}$ & 0.0001 \\
\hline TNF- $\alpha$ & $\begin{array}{c}\mathbf{0 . 2 5} \\
0.00,4.3\end{array}$ & $\begin{array}{c}5.29 \\
0.00,13.06\end{array}$ & 0.0071 \\
\hline LT- $\alpha$ & $\begin{array}{c}0.00 \\
0.00,0.00\end{array}$ & $\begin{array}{c}0.00 \\
0.00,5.855\end{array}$ & 0.0031 \\
\hline IFN-y & $\begin{array}{c}\mathbf{0 . 0 0} \\
0.00,30.585\end{array}$ & $\begin{array}{c}28.34 \\
0.00,67.59\end{array}$ & 0.0037 \\
\hline IFN- $\alpha$ & $\begin{array}{c}0.00 \\
0.00,0.025\end{array}$ & $\begin{array}{c}0.00 \\
0.00,7.395\end{array}$ & 0.0140 \\
\hline IL-17 & $\begin{array}{l}190.9167 \\
128,269.5\end{array}$ & $\begin{array}{c}209.4167 \\
142.9167,299.2925\end{array}$ & 0.4056 \\
\hline IL-12p70 & $\begin{array}{c}\mathbf{0 . 0 0} \\
0.00,2.045\end{array}$ & $\begin{array}{c}0.00 \\
0.00,5.055\end{array}$ & 0.0516 \\
\hline
\end{tabular}

B

\begin{tabular}{|c|c|c|c|c|c|}
\hline Cytokine & Minimal & Mild & Moderate & Severe & p Value \\
\hline IP-10 & $\begin{array}{c}725.78 \\
520.89,1079.83\end{array}$ & $\begin{array}{c}759.69 \\
571.805,1139.695\end{array}$ & $\begin{array}{c}749.52 \\
412.445,946.965\end{array}$ & $\begin{array}{c}636.66 \\
433.035,941.62\end{array}$ & 0.5984 \\
\hline TNF- $\alpha$ & $\begin{array}{c}8.83 \\
0.00,29.98\end{array}$ & $\begin{array}{c}7.93 \\
0.00,22.23\end{array}$ & $\begin{array}{c}0.00 \\
0.00,7.49\end{array}$ & $\begin{array}{c}4.38 \\
0.00,8.195\end{array}$ & 0.0214 \\
\hline LT- $\alpha$ & $\begin{array}{c}1.11 \\
0.00,17.535\end{array}$ & $\begin{array}{c}0.00 \\
0.00,11.46\end{array}$ & $\begin{array}{c}0.00 \\
0.00,1.135\end{array}$ & $\begin{array}{c}0.00 \\
0.00,2.53\end{array}$ & 0.0426 \\
\hline IFN- $\gamma$ & $\begin{array}{c}28.39 \\
0.00,80.43\end{array}$ & $\begin{array}{c}36.18 \\
0.00,82.39\end{array}$ & $\begin{array}{c}31.73 \\
0.00,58.11\end{array}$ & $\begin{array}{c}18.85 \\
0.00,65.32\end{array}$ & 0.9526 \\
\hline IFN- $\alpha$ & $\begin{array}{c}0.00 \\
0.00,10.685\end{array}$ & $\begin{array}{c}0.00 \\
0.00,10.15\end{array}$ & $\begin{array}{c}0.00 \\
0.00,2.82\end{array}$ & $\begin{array}{c}0.00 \\
0.00,3.98\end{array}$ & 0.1156 \\
\hline IL-17 & $\begin{array}{c}194.5 \\
129.9325,301.5\end{array}$ & $\begin{array}{c}206.25 \\
149.375,289.5\end{array}$ & $\begin{array}{c}204.8333 \\
157.5,313.75\end{array}$ & $\begin{array}{l}223.8333 \\
153,341.5\end{array}$ & 0.5559 \\
\hline IL-12p70 & $\begin{array}{c}4.99 \\
2.98,6.5\end{array}$ & $\begin{array}{c}0.00 \\
0.00,3.565\end{array}$ & $\begin{array}{c}0.00 \\
0.00,4.16\end{array}$ & $\begin{array}{c}0.00 \\
0.00,3.78\end{array}$ & 0.0608 \\
\hline
\end{tabular}


4Fig. 1 a Table of proinflammatory cytokine levels in patients with pSS. Values in the table represent median and 25th, 75th centile (pmol/L). Bold typeface indicates statistical significance of cytokine serum level between fatigue groups as determined by ANOVA. b Proinflammatory cytokine levels in patients with pSS. Values in the table represent median and $25 \mathrm{th}, 75$ th centile $(\mathrm{pmol} / \mathrm{L})$. Bold typeface indicates statistical significance of cytokine serum level between fatigue groups as determined by ANOVA

\section{Proinflammatory cytokines and fatigue severity in pSS}

Given the replication of an inverse relationship between proinflammatory cytokines and fatigue in pSS patients, we ran an additional analysis as in the original paper [8]. This logistic regression model was originally used to establish whether fatigue levels could be identified using cytokines, disease-specific and clinical parameters as well as pain, anxiety, and depression. We repeated this analysis by initially examining whether cytokines and/or covariates could successfully identify fatigue category. We then looked at the effect of each cytokine on the model. The full model, including all seven cytokines, was able to correctly identify fatigue category in $85 \%$ of cases (Fig. 2). This model with all parameters was sensitive to the presence or absence of cytokines, depression, anxiety, and pain, but was robust to the presence or absence of other markers of disease activity (WCC, neutrophils, ESR, CRP, ESSDAI, and dryness). We ran a reduced model including the covariates depression, anxiety, and pain alongside LT- $\alpha$ and TNF- $\alpha$. The reduced model correctly identified fatigue category in $85 \%$ of cases, comparable to the full model. The cytokines IP-10, LT- $\alpha$, IFN- $\gamma$, and IL-17 demonstrated the same inverse relationship with fatigue category.

\section{Discussion}

Our study confirms that pSS patients with higher levels of fatigue had lower levels of the proinflammatory cytokines than patients with pSS with lower levels of fatigue. This inverse relationship was observed for all four of the original cytokines, though it was statistically significant only for LT- $\alpha$ and TNF- $\alpha$.

LT- $\alpha$ and TNF- $\alpha$ are closely related cytokines and are both produced by an activated Th1 response. Howard-Tripp et al. discussed the possibility that dysregulation of Th1 responses could be affiliated with the development of fatigue with the potential that $\mathrm{Th} 2$ responses could be important in maintaining fatigue, as demonstrated in CFS [17]. The cytokine abnormalities in CFS, however, have been inconsistent, possibly complicated by confounding psychosocial factors.
There is a documented association between Type 1 IFN signature, often implicated in the pathogenesis of pSS, and TNF- $\alpha$ in autoimmune disease [18]. Data from our research group, however, has recently demonstrated no association with IFN- $\alpha$ or Type I IFN signature with fatigue in pSS [19]. Equally, our findings of the relationship between TNF- $\alpha$ and fatigue would explain the failure in fatigue reduction in patients treated with anti-TNF- $\alpha$ medications [20].

Taken together, our data from this and previous study do not support the simple concept of higher levels of inflammation leading to worse fatigue. Instead, we propose that regulatory mechanisms of inflammation may be responsible for the maintenance of fatigue after the initial proinflammatory response. The presence of persistent immune challenge results in chronic inflammation, which may triggers an inappropriate or exaggerated anti-inflammatory response. Such anti-inflammatory mechanisms may in turn have a role in the persistence of fatigue. In other words, we postulated that the exaggerated or inappropriate immune regulation turns what was an adaptive behavioural response, sickness behaviour, into a persistent, pathological response resulting in chronic fatigue with or without concomitant pain and depression. Identifying such anti-inflammatory mechanisms may give clue to treatment of fatigue in pSS.

The strengths of this validation study include (1) a large, clinically well-defined patient group; (2) minimal demographic variation between fatigue groups in the pSS cohort; (3) the use of validated measures in pSS; and (4) consistency of methods with the original study.

Limitations of this study include the (1) cross-sectional nature of this study; (2) fluctuations of cytokine levels could be influenced by multiple factors; (3) while the original findings have been validated in an independent cohort taken from the UKPSSR, a full external validation in another cohort is needed; and (4) using an all-female cohort, similar to the original study, while minimising the potential gender differences in cytokine profile, means our findings may not be generalisable to men with $\mathrm{pSS}$.

\section{Conclusion}

This study has validated our previous observation of an inverse relationship between proinflammatory cytokines and fatigue levels in pSS. Our data further challenges the notion that proinflammatory cytokines directly mediate fatigue in pSS. Further studies to determine whether the model also applies to other chronic immune-mediated inflammatory conditions and to explore the possible mechanisms of this inverse relationship between proinflammatory cytokines and fatigue levels are warranted. 


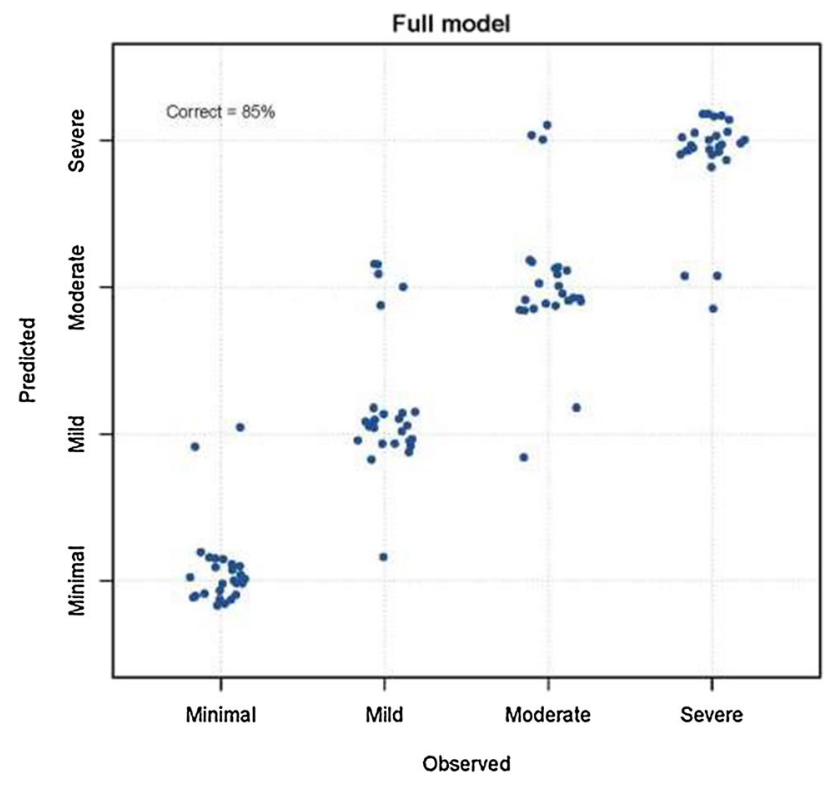

Fig. 2 Observed and predicted fatigue levels for the ordinal logistic regression model with all seven cytokines, WCC, lymphocytes, neutrophils, ESR, CRP, ESSDAI scores, dryness scores, depression, pain, and anxiety. This full model predicts fatigue level correctly in $85 \%$ of cases

Acknowledgements WFN, SJB, and BG are investigators of the UKPSSR. The other UKPSSR members (as of 1 Jan 2017) include in alphabetical order of their affiliations: Frances Hall (Addenbrooke's Hospital, Cambridge); Elalaine C Bacabac, Helen Frankland, Robert Moots (Aintree University Hospitals); Kuntal Chadravarty, Shamin Lamabadusuriya (Barking, Havering and Redbridge NHS Trust); Michele Bombardieri, Constantino Pitzalis, Nurhan Sutcliffe, Celia Breston (Bart and the London NHS Trust); Nagui Gendi, Karen Culfear (Basildon Hospital); Claire Riddell (Belfast Health and Social Care Trust); John Hamburger, Andrea Richards (Birmingham Dental Hospital); Saaeha Rauz (Birmingham \& Midland Eye Centre); Sue Brailsford, Joanne Dasgin (Birmingham University Hospital); Joanne Logan, Diarmuid Mulherin (Cannock Chase Hospital); Jacqueline Andrews, Paul Emery, Alison McManus, Colin Pease, David Pickles (Chapel Allerton Hospital, Leeds); Alison Booth, Marian Regan (Derbyshire Royal Infirmary); Jon King, Amanda Holt (Derriford Hospital, Plymouth); Theodoros Dimitroulas, Lucy Kadiki, Daljit Kaur, George Kitas (Dudley Group of Hospitals NHS Foundation Trust); Abdul Khan, Tracey Cosier (East Kent Hospitals University); Panthakalam, Kelly Mintrim (East Sussex Healthcare NHS); Mark Lloyd, Lisa Moore (Frimley Park Hospital); Esther Gordon, Cathy Lawson (Harrogate District Foundation Trust Hospital); Monica Gupta, John Hunter, Lesley Stirton (Gartnavel General Hospital, Glasgow); Gill Ortiz, Elizabeth Price; Suzannah Pelger (Great Western Hospital); Claire Gorman, Balinder Hans (Homerton Hospital); Gavin Clunie, Suzanne Lane, Ginny Rose, Sue Cuckow (Ipswich Hospital NHS Trust); Michael Batley, Ruby Einosas (Maidstone Hospital); Susan Knight, Deborah Symmons, Beverley Jones (Macclesfield District General Hospital \& Arthritis Research UK Epidemiology Unit, Manchester); Andrew Carr, Suzanne Edgar, Francisco Figuereido, Heather Foggo, Dennis Lendrem, Iain Macleod, Sheryl Mitchell, Christine Downie, Jessica Tarn, James Locke, Shereen Al-Ali, Sarah Legg, Kamran Mirza, Ben Hargreaves, Laura Hetherington (Newcastle upon Tyne Hospitals NHS Foundation Trust and Newcastle University); Adrian Jones, Peter
Lanyon, Alice Muir (Nottingham University Hospital); Paula White, Steven Young-Min (Portsmouth Hospitals NHS Trust); Susan Pugmire, Saravanan Vadivelu (Queen's Elizabeth Hospital, Gateshead); Annie Cooper, Marianne Watkins (Royal Hampshire County Hospital); Anne Field, Stephen Kaye, Devesh Mewar, Patricia Medcalf, Pamela Tomlinson, Debbie Whiteside (Royal Liverpool University Hospital); Neil McHugh, John Pauling, Julie James, Andrea Dowden (Royal National Hospital for Rheumatic Diseases); Mohammed Akil, Jayne McDermott, Olivia Godia (Royal Sheffield Hospital); David Coady, Elizabeth Kidd, Lynne Palmer (Royal Sunderland Hospital); Charles Li (Royal Surrey Hospital); Sarah Bartrum, Dee Mead (Salisbury District Hospital); Bhaskar Dasgupta, Victoria Katsande, Pamela Long, Olivia Godia (Southend University Hospital); Erin Vermaak, Janet Turner (Staffordshire Stoke-on-Trent Partnership); Usha Chandra, Kirsten MacKay (Torbay Hospital); Stefano Fedele, Ada Ferenkeh-Koroma, Ian Giles, David Isenberg, Helena MaConnell, Nyarko Ahwireng, Stephen Porter (University College Hospital \& Eastman Dental Institute); Paul Allcoat, John McLaren (Whyteman's Brae Hospital, Kirkcaldy).

Authorship This work was drafted by W-FN, SJB, DL, JT, and NHT and designed by W-FN, JT, KD, and KM. Experimentation and analysis completed by KD, KM, and JT. KD and KM drafted the original manuscript. DT, SJB, NHT, and WFN provided revisions. All authors approved the final version and are in agreement to be accountable for all aspects of work.

Funding This study received grant support from the Medical Research Council, UK (G0800629 to W-FN, SJB) for the creation of the UKPSSR. This work also received infrastructure support from the National Institute for Health Research Newcastle Biomedical Research Centre based at Newcastle Hospitals NHS Foundation Trust and Newcastle University. The views expressed are those of the author(s) and not necessarily those of the NHS, the NIHR or the Department of Health.

\section{Compliance with ethical standards}

Conflict of interest K. Davies declares that he has no conflicts of interest. K. Mirza declares that he has no conflicts of interest. J. Tarn declares that she has no conflicts of interest. N. Howard-Tripp declares that she has no conflicts of interest. S J Bowman declares that he has no conflicts of interest. D. Lendrem declares that he has no conflicts of interest. W.-F. Ng declares that he has no conflicts of interest

Research involving human participants and/or animals Patient and control samples were provided from the biobank of the United Kingdom Primary Sjögren's Syndrome Registry (UKPSSR), which is funded by the Medical Research Council and based in Newcastle. Ethical approval for the use of human samples was granted by the North West Research Ethics Committee. The procedures used to analyse these samples followed were in accordance with the ethical standards of the North West Research Ethics Committee with the Helsinki Declaration 2013.

Informed consent Informed consent was gained from all patients and controls who have submitted their serum to the UKPSSR biobank for its use in research and for publication of its results.

Open Access This article is distributed under the terms of the Creative Commons Attribution 4.0 International License (http://creativeco mmons.org/licenses/by/4.0/), which permits unrestricted use, distribution, and reproduction in any medium, provided you give appropriate credit to the original author(s) and the source, provide a link to the Creative Commons license, and indicate if changes were made. 


\section{References}

1. Cullen W, Kearney Y (2002) Bury G (2002) Prevalence of fatigue in general practice. Ir J Med Sci 171(1):10-12

2. Bultmann U, Kant I, Kasl SV, Beurskens AJ, van den Brandt PA (2002) Fatigue and psychological distress in the working population: psychometrics, prevalence, and correlates. J Psychosom Res 52(6):445-452

3. Lerdal A, Wahl A, Rustoen T, Hanestad BR, Moum T (2005) Fatigue in the general population: a translation and test of the psychometric properties of the Norwegian version of the fatigue severity scale. Scand J Public Health 33(2):123-130

4. Reynolds KJ, Vernon SD, Bouchery E, Reeves WC (2004) The economic impact of chronic fatigue syndrome. Cost Eff Resour Alloc 2004:2. https://doi.org/10.1186/1478-7547-2-4

5. Meijer JM, Meiners PM, Huddleston Slater JJ, Spijkervet FK, Kallenberg CG, Vissink A et al (2009) Health-related quality of life, employment and disability in patients with Sjogren's syndrome. Rheumatology (Oxford, England) 48(9):1077-1082

6. Haldorsen K, Bjelland I, Bolstad AI, Jonsson R, Brun JG (2011) A five-year prospective study of fatigue in primary Sjögren's syndrome. Arthritis Res Ther 13(5):R167

7. Arnett SV, Clark IA (2012) Inflammatory fatigue and sickness behaviour-lessons for the diagnosis and management of chronic fatigue syndrome. J Affect Disord 141(2-3):130-142

8. Howard Tripp N, Tarn J, Natasari A, Gillespie C, Mitchell S, Hackett KL et al (2016) Fatigue in primary Sjögren's syndrome is associated with lower levels of proinflammatory cytokines. RMD Open 2(2):e000282

9. Ng WF, Bowman SJ (2010) Primary Sjogren's syndrome: too dry and too tired. Rheumatology (Oxford, England) 49(5):844-853

10. Ng W-F, Bowman SJ, Griffiths B (2011) United Kingdom Primary Sjögren's Syndrome Registry - a united effort to tackle an orphan rheumatic disease. Rheumatology 50(1):32-39

11. Neuberger GB (2003) Measures of fatigue: the Fatigue Questionnaire, Fatigue Severity Scale, Multidimensional Assessment of Fatigue Scale, and Short Form-36 Vitality (Energy/Fatigue)
Subscale of the Short Form Health Survey. Arthritis Care Res 49(S5):S175-S183

12. Bjelland I, Dahl AA, Haug TT, Neckelmann D (2002) The validity of the Hospital Anxiety and Depression Scale. An updated literature review. J Psychosom Res 52(2):69-77

13. Seror R, Ravaud P, Bowman SJ, Baron G, Tzioufas A, Theander E et al (2010) EULAR Sjögren's syndrome disease activity index: development of a consensus systemic disease activity index for primary Sjögren's syndrome. Ann Rheum Dis 69(6):1103-1109

14. Seror R, Ravaud P, Mariette X, Bootsma H, Theander E, Hansen A et al (2011) EULAR Sjögren's Syndrome Patient Reported Index (ESSPRI): development of a consensus patient index for primary Sjögren's syndrome. Ann Rheum Dis 70(6):968-972

15. SAS (2019) JMP statistical discovery software. http://www.jmp. com/en_gb/home.html. Accessed 25 June 2019

16. The R Foundation (2019) R project for statistical computing. https ://www.r-project.org/. Accessed 25 June 2019

17. Skowera A, Cleare A, Blair D, Bevis L, Wessely SC, Peakman M (2004) High levels of type 2 cytokine-producing cells in chronic fatigue syndrome. Clin Exp Immunol 2004:135

18. Palucka AK, Blanck J-P, Bennett L, Pascual V, Banchereau J (2005) Cross-regulation of TNF and IFN- $\alpha$ in autoimmune diseases. Proc Natl Acad Sci USA 102(9):3372-3377

19. Bodewes ILA, Al-Ali S, van Helden-Meeuwsen CG, Maria NI, Tarn J, Lendrem DW et al (2018) Systemic interferon type I and type II signatures in primary Sjögren's syndrome reveal differences in biological disease activity. Rheumatology 57(5):921-930

20. Mariette X, Ravaud P, Steinfeld S, Baron G, Goetz J, Hachulla $E$ et al (2004) Inefficacy of infliximab in primary Sjögren's syndrome: results of the randomized, controlled trial of remicade in primary Sjögren's syndrome (TRIPSS. Arthritis Rheum 50(4):1270-1276

Publisher's Note Springer Nature remains neutral with regard to jurisdictional claims in published maps and institutional affiliations.

\section{Affiliations}

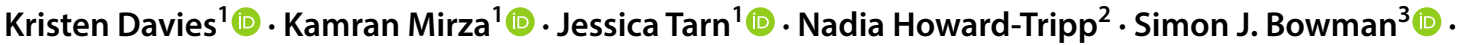 Dennis Lendrem ${ }^{1,2,4} \odot$. UK Primary Sjögren's Syndrome Registry $\cdot$ Wan-Fai $\mathrm{Ng}^{1,2,4}(\mathbb{C}$}

\author{
Kamran Mirza \\ kamran.mirza@newcastle.ac.uk \\ Jessica Tarn \\ jessica.tarn@newcastle.ac.uk \\ Nadia Howard-Tripp \\ nadia.howard-tripp@newcastle.ac.uk \\ Simon J. Bowman \\ Simon.Bowman@uhb.nhs.uk \\ Dennis Lendrem \\ dennis.lendrem@newcastle.ac.uk \\ Wan-Fai Ng \\ wan-fai.ng@newcastle.ac.uk
}

1 Musculoskeletal Research Group, Institute of Cellular Medicine, Newcastle University, Newcastle upon Tyne NE2 4HH, UK

2 Newcastle-upon-Tyne Hospitals NHS Foundation Trust, Newcastle upon Tyne, UK

3 University Hospitals Birmingham NHS Foundation Trust, Birmingham, UK

4 National Institute of Health Research Biomedical Research Centre, Newcastle University, Newcastle upon Tyne, UK 